\title{
Strategy for Compositional Analysis of the Hair Cell Mechanotransduction Complex Using TIRF Microscopy
}

\author{
Sarah Clark ${ }^{1}$, Johannes Elferich ${ }^{1}$, Jingpeng Gai ${ }^{1}$, April Goehring ${ }^{1}$, Jaba Mitra ${ }^{2}$, Taekjip Ha ${ }^{2,3}$ and Eric \\ Gouaux ${ }^{1,4^{*}}$ \\ 1. Vollum Institute, Oregon Health \& Science University, Portland, United States. \\ 2. Department of Biophysics and Biophysical Chemistry, Department of Biophysics, Department of \\ Biomedical Engineering, Johns Hopkins University, Baltimore, United States. \\ 3. Howard Hughes Medical Institute, Johns Hopkins University, Baltimore, United States. \\ 4. Howard Hughes Medical Institute, Oregon Health \& Science University, Portland, United States. \\ * Corresponding author: gouauxe@ohsu.edu
}

Mechanotransduction machinery located at the tips of hair bundles in the inner ear are responsible for our sensations of movement and sound. Each bundle is composed of $\sim 100$ stereocilia organized in a staircase array that are connected by tip-links, extracellular protein filaments composed of protocadherin 15 (PCDH15) and cadherin-23 (CDH23) [1]. Deflection of the hair bundle by sound and fluid movement leads to opening of the mechanotransduction channel (MET) complex located at the lower tip-link insertion site, resulting in an electrical signal. There are three putative components of the MET complex aside from the tip-link proteins: the lipoma HMGIC fusion partner-like 5 protein LHFPL5 (also known as TMHS) $[2,3]$, the transmembrane inner ear protein TMIE, and the transmembrane-like channel proteins $\mathrm{TMC} 1 / 2[4,5]$, which are the likely pore-forming subunits of the complex [6]. It is estimated that there is only one MET complex per stereocilia, which means there is approximately 1 attomole of complex per mouse cochlea [6]. This low abundance of material cannot be detected by conventional methods of analysis, such as ELISA or western blot.

Here we present a strategy to overcome this limitation and analyze the MET complex from mouse cochlea: single molecule pulldown (SiMPull). SiMPull combines a conventional pull-down assay with single molecule total internal reflection (TIRF) microscopy to examine protein complexes from cell or tissue extracts [7]. An antibody immobilized on a coverslip is used to capture the MET complex from solution, and another antibody or antibody fragment (Fab) linked to a fluorophore enables visualization of the complex (Figure 1A). This approach enables the study of individual molecules, thus allowing analysis of the composition and stoichiometry of a low abundance complex.

We have successfully developed an ultrasensitive SiMPull assay using recombinantly expressed PCDH15/LHFPL5 complex. We have generated several high affinity monoclonal antibodies (mAbs) against PCDH15 and verified that they recognize native protein in mouse cochlea via immunofluorescence. Using these mAbs, we are able to reliably detect as low as 12 attomoles of PCDH15/LHFPL5 complex (Figure 1B), well within the sensitivity needed to study native protein. We have also established a method to measure the stoichiometry of PCDH15 by photobleaching particles bound to engineered Fabs with a GFP tag. The results indicate that PCDH15 is dimeric (Figure 1C), in agreement with the cryo-EM structure of PCDH15/LHFPL5 [8].

The SiMPull assay has the potential to reveal important compositional information about the native MET complex. In addition to our anti-PCDH15 mAbs, we have developed antibodies against TMC1 and TMIE that can be used to probe the composition of the native complex extracted from mouse cochlea. Pulling 
down with an anti-PCDH15 mAb and detecting with anti-TMC1 and anti-TMIE mAbs, for example, would demonstrate the existence of an intact complex and allow us to measure the stoichiometry of these proteins bound to PCDH15. While many previous studies have shown in vivo subunit interaction by localization to the stereocilia tip or colocalization in cells $[2,3,9,10]$, these studies demonstrate that the proteins are near each other, but not necessarily within the same complex.

References:

[1] P Kazmierczak et al., Nature 449 (2007), p. 87.

[2] M Beurg et al., PNAS 112 (2015), p. 1589.

[3] W Xiong et al., Cell 151 (2012), p. 1283.

[4] K Kurima et al., Cell reports 12 (2015), p. 1606.

[5] B Pan et al., Neuron 79 (2013), p. 504.

[6] R Fettiplace, Biophysical journal 111 (2016), p. 3.

[7] A Jain et al., Nat Protoc 7 (2012), p. 445.

[8] JE Ge et al., eLife 7 (2018).

[9] B Zhao et al., Neuron 84 (2014), p. 954.

[10] R Maeda et al., PNAS 111 (2014), p. 12907.

A
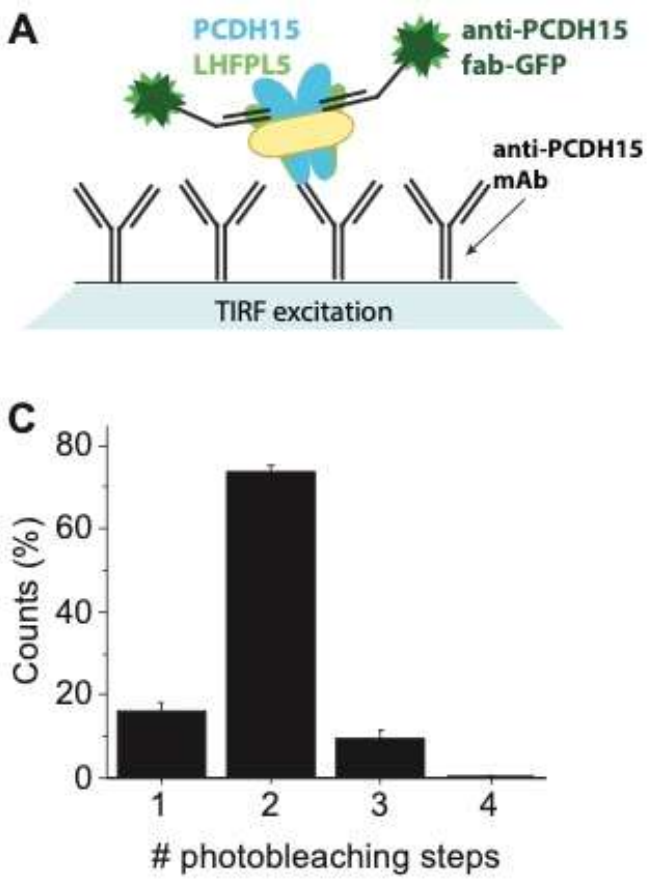

B

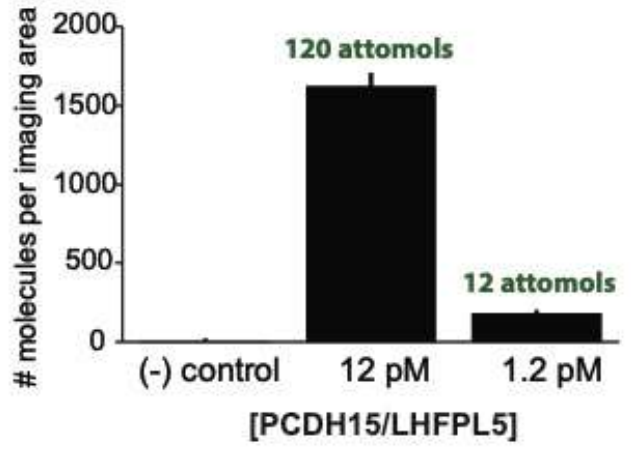

Figure 1. (A) SiMPull schematic showing capture of the recombinant PCDH15/LHFPL5 complex with anti-PCDH15 Fab and detection with anti-PCDH15 mAb. (B) Bar graph with average number of fluorphores per image for PCDH15/LHFPL5 complex at different concentrations. The negative control channel uses a control antibody for capture. (C) Bar graph showing photobleaching of PCDH15/LHFPL5 using GFP-tagged Fab. 